\title{
Joint routing and scheduling in multi-channel capillary machine-to-machine networks
}

\author{
Taeshik Shon ${ }^{1}$, Hyo Hyun $\mathrm{Choi}^{2}$ and Eui-Jik Kim ${ }^{3 *}$
}

\begin{abstract}
In recent times, machine-to-machine (M2M) communication is considered the most promising solution for revolutionizing future intelligent pervasive applications. The capillary M2M network is a key component of the M2M system, and it should support a data rate that is sufficient to accommodate various vertical applications with limited resources of end devices. This paper presents a joint routing and scheduling (JRS) framework for a capillary M2M network. To achieve the conflicting goals of maximizing network performance and conserving energy, the JRS framework is designed with an integrated concept of routing and transmission scheduling, which provides time division multiple access (TDMA)-based transmission scheduling based on the established routing path of data. Moreover, to extend the number of available transmission frames and enhance network performance, JRS uses the multi-channel communication approach. Experimental results of the prototype implementation demonstrate that JRS outperforms conventional schemes, even under heavy traffic conditions.
\end{abstract}

Keywords: Capillary machine-to-machine, Prototype implementation, Routing, Transmission scheduling

\section{Introduction}

In recent times, machine-to-machine (M2M) communication has emerged as a cutting-edge technology for next-generation communication, and it is considered the most promising solution for revolutionizing future intelligent pervasive applications [1-6]. Wireless M2M communication is a form of data transfer that facilitates direct communication among machines with little or no human intervention. Recently, considerable effort has been put into M2M standardization [7,8]. For example, the European Telecommunications Standards Institute has launched the M2M Technical Committee with the purpose of developing an end-to-end architecture for M2M communications [9] in which the capillary M2M network is considered a key component of the M2M system. A capillary M2M network is a generic term that refers to any network technology that provides physical and medium access control (MAC) layer connectivity between various M2M devices connected to the same capillary M2M network or that allows an M2M device to gain access to a public network via a router or a

\footnotetext{
* Correspondence: euijik.kim@kt.com

${ }^{3}$ Advanced Institute of Technology, KT Corporation, Umyeon-dong, Seocho-gu, Seoul 137-792, South Korea

Full list of author information is available at the end of the article
}

gateway. In [10], it is also referred to as an M2M area network.

Capillary M2M networks should support a data rate that is sufficient to accommodate various kinds of vertical applications with the limited resources of end devices. In other words, to deliver various kinds and sizes of M2M data effectively, capillary M2M networks must be able to both maximize the network throughput and conserve energy. Here, we discuss several issues that need to be considered in the design of a capillary M2M software framework to meet the above requirements.

The first issue pertains to the MAC protocol, which plays a key role in determining the network throughput, delay, power consumption, etc. A large number of MAC protocols have been proposed for sensor networks and personal area networks, with energy efficiency as the primary design criterion. The most common of these protocols are the contention-based carrier sense multiple access (CSMA) scheme [11-17] and the schedulebased time division multiple access (TDMA) scheme [18-20]. CSMA is the natural solution because of its simplicity and flexibility. With the CSMA-based MAC protocol, individual devices in a network can decide how and when to access the radio medium without any time synchronization or topology information. However, their 
performance can be significantly degraded in situations of high contention because of the high overhead accrued for resolving collisions. In addition, overhearing and idle listening also affect energy consumption. In contrast, TDMA utilizes time synchronization among neighboring devices to achieve collision-free transmission by assigning unique transmission time slots to individual devices. TDMA can generally achieve higher network throughput in situations of high contention, as well as energy efficiency. However, scheduling the time slots is notoriously difficult to achieve in a distributed fashion, and strict synchronization is also required. In addition, TDMA often suffers from inefficiency in low contention situations, because a device can transmit only during its scheduled time slots. Hu et al. [21] reported that while CSMA suffers from inefficiency in high contention situations, TDMA operates in a converse manner. Therefore, the strengths of both types of MAC protocols must be combined while offsetting their shortcomings.

The second issue concerns the routing path of data from end devices to an M2M gateway. Network design has traditionally followed the principle of layering. Previous work in this area has focused largely on the design of link-level protocols [22-28], and some previous papers address routing for such a network $[29,30]$. However, the formation of the network topology affects the operation of the MAC protocol, which in turn, significantly affects the performance factors, such as network throughput and energy consumption. Thus, the network layer must be designed to include a routing protocol in conjunction with diverse MAC-related scheduling.

In this paper, we propose a joint routing and scheduling (JRS) architecture in a capillary M2M network that seeks to address all the aforementioned issues. The contributions of our work are twofold. First, we designed the JRS architecture with the following attractive features: (1) The CSMA-TDMA hybrid MAC approach combines the strengths of both types of MAC protocols while offsetting their shortcomings. CSMA-TDMA hybrid MAC features scalability and robustness using CSMA/collision avoidance and provides considerable guaranteed and collision-free transmission of critical data using TDMA. (2) Integrated routing and MAC scheduling minimizes the protocol overhead. JRS organizes the multi-hop tree network through a route management procedure and assigns the subframe to each end device in a distributed manner. (3) Multi-channel communication extends the number of subframes and enhances network performance. Second, we provide an actual implementation and build a prototype employing the proposed framework, for the proof-of-concept and performance evaluations. Most of the aforementioned network protocols for capillary M2M networks have been evaluated only through simulation experiments. To validate the practicality and feasibility of the proposed framework, we implemented JRS in the TinyOS 2.0 operating system [31] and compared it with the ZigBee solution using self-developed hardware prototype devices. We show that JRS outperforms ZigBee in terms of the average packet reception rate, average per-hop latency, and energy efficiency.

The remainder of this paper is organized as follows: in JRS network architecture, we present the overall JRS network architecture. JRS operation describes the operation of JRS. Prototype implementation details a practical implementation through which we evaluate the JRS framework through extensive experiments. We conclude the paper in the 'Conclusion' section.

\section{JRS network architecture}

JRS operates as a network layer subsystem on the entire networking stack and interacts with neighboring layers, namely the lower MAC layer in the bottom and upper application layer on the top. The JRS subsystem jointly optimizes routing and scheduling in a multi-channel multi-hop network of the M2M domain. Figure 1 shows the JRS network architecture, which consists of the following four function blocks: route management (RM), subframe management (SFM), join and route recovery (JRR), and received message handler (RMH).

The RM block has three main functions: first, route advertisement is for exchanging route information among the devices. Using this function, the devices can discover their neighbors. Second, join is for selecting a parent device and requesting to join it. Finally, slot allocation is for assigning a TDMA slot within the subframe to the children. Slot allocation is performed when a device receives a join request (JREQ) from a child candidate device. The SFM block has two main functions: subframe advertisement is for exchanging the subframe information among the devices, by which each device can maintain channel occupancy status of its neighbors within the interference range. Subframe collision resolution is for avoiding the selection of a duplicate subframe. When two or more devices within interference range select the same subframe, this function is performed. The JRR block has two main functions: neighbor discovery is for collecting the neighbor's information using a HELLO message when the device is newly turned on or detects a link error. Link status detection is for detecting the occurrence of a link failure. The RMH block is for classifying and processing received packets. According to the kind of packet received, RMH calls the appropriate function. In addition, the JRS subsystem maintains multiple data structures, namely JRS information base (JRSIB), which includes a routing table, a subframe table, and a child table. The aforementioned function blocks interact with these tables. In the 


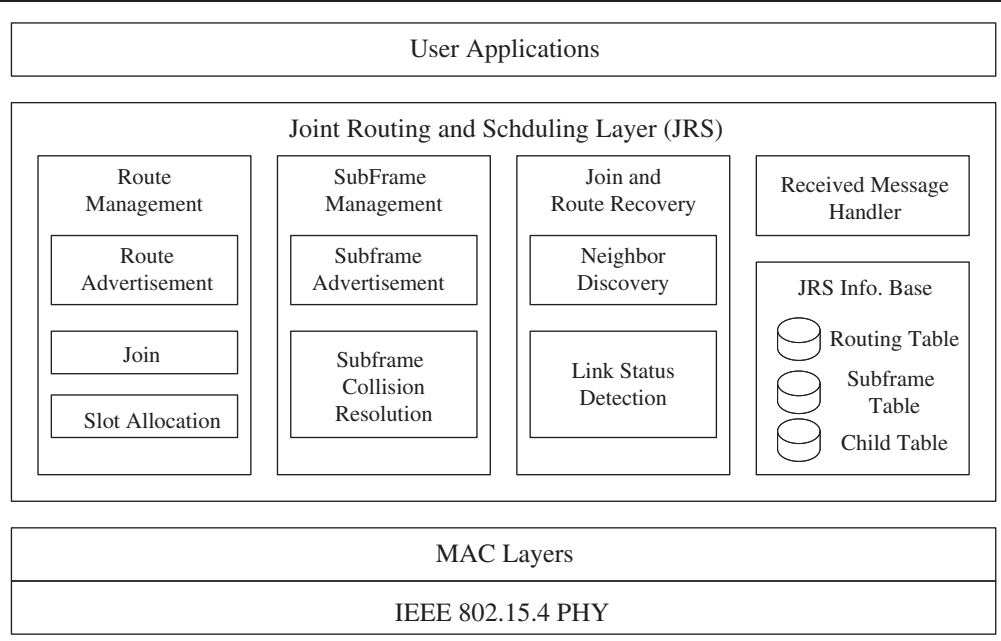

Figure 1 Network architecture of JRS.

following sections, we present detailed descriptions of each block.

\section{JRS operation}

In this paper, we focus on the development and implementation of the unattended environmental monitoring M2M system, in which the end device has a stringent energy requirement. Consequently, in order to maximize the lifetime of the capillary M2M network, we use TDMA-based on/off scheduling as a baseline MAC scheme. Moreover, to enhance network performance and avoid collisions in the neighboring area, we also consider the use of multi-channel communication. As a network starts up, JRS first sets up the tree shape of routes and allocates channels and time slots needed for TDMA-based operation, using CSMA. After completing these procedures, all the devices in the network operate in TDMA mode according to their fixed schedules at the exact time. JRS basically performs the route setup and the subframe allocation. In addition, it provides the joining-support functionality for newly turned-on devices en route. JRS also performs route recovery when a route failure is detected. Assuming that the clocks of all the network devices are synchronized exactly (i.e., every device has to maintain the same timer), the M2M gateway starts the route setup procedure.

Figure 2 shows the overall operation of JSR. In the setup phase, all the devices in the network operate in CSMA mode using a predefined common channel and perform the route setup and subframe allocation. In the figure, the time is divided into two phases. During the setup phase, JRS establishes the route from each end device to the M2M gateway device in the tree- topology shape. Based on the established routing path, JRS allocates a subframe to each device. After the subframe allocation is completed, each device can maintain its own subframe table for k-hop distance, which is the interference range of the device and in which the numerical value of $\mathrm{k}$ has to be defined through the experiment. In addition, the communication between a pair of devices is always performed at the parent's subframe.

If the status of network becomes stable (i.e., the route and the subframe of each device do not change) during the setup phase, the devices in the network have to operate in TDMA mode according to the predefined active/sleep schedule (TDMA-based on/off phase). In other words, the communication schedule of all the devices including transmit, receive, and sleep is fixed. In the TDMA region, the time is divided into a large period $T$, and the period, $T$, is further divided into $N$ small periods, as shown in Figure 2. This small period, $T / N$, is the unit of the subframe used by each device.

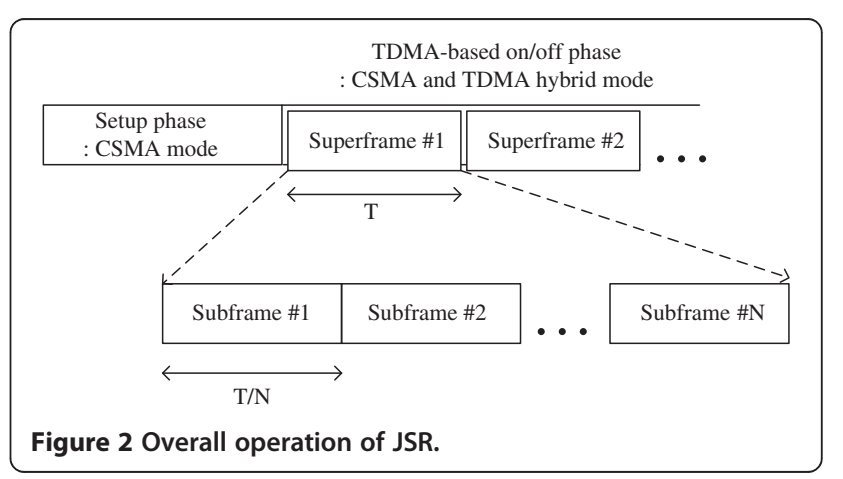


As mentioned earlier, JRS maintains the four function blocks (RM, SFM, JRR, and RMH) for which various JRS control messages are exchanged among the devices. These control messages include the following: route advertisement (RADV), JREQ, join confirm (JCNF), channel advertisement (CADV), HELLO, route information request (RREQ), and route and subframe information request (RSREQ).

\subsection{Route management}

During the setup phase (operating in CSMA mode), route management is enabled. Figure 3 shows an example of route management. As a device starts up, it first runs a route advertisement (i.e., neighbor discovery) procedure to create the routing table, whose entries include the device ID, destination ID (gateway device ID), next-hop ID, hop count to the gateway, and parent/child relationship. Each device maintains entries only for those neighbors within the k-hop distance (i.e., interference range). This information is used for the subsequent tree route construction. The route advertisement procedure works in a manner similar to distance vector (DV) algorithms in that the gateway creates the RADV message including its routing table entry and broadcasts it to each of its neighbors, and the neighbors that receive this RADV update their routing table and rebroadcast it in sequence.

To set up the shortest path to the gateway, each device in the network waits until the routing table entries become stable as this procedure is repeated. After the routing table stabilizes, the device runs join and slot allocation procedures by sending the JOIN message to the parent. When the parent receives this message, it registers a corresponding device to its child table and responds with the JCNF message. Through the JCNF message, the child device is evenly assigned with time slots within a subframe to exchange data. The time slot

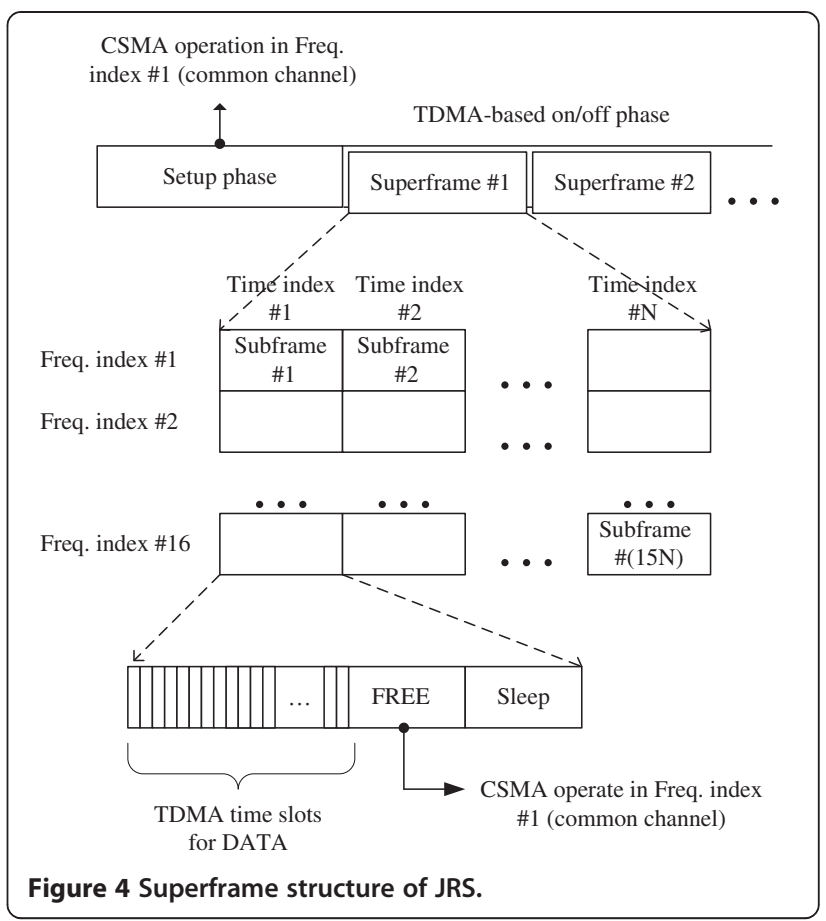

structure within a subframe is described in the next subsection.

\subsection{Subframe management}

Route management and subframe management procedures are performed nearly simultaneously. In the subframe management procedure, each device in the network is assigned a channel (i.e., subframe) for communication between a parent-child pair. Figure 4 shows the superframe structure of the JRS framework. In the figure, the time is divided into superframes, each superframe is divided into $N$ subframes, and each

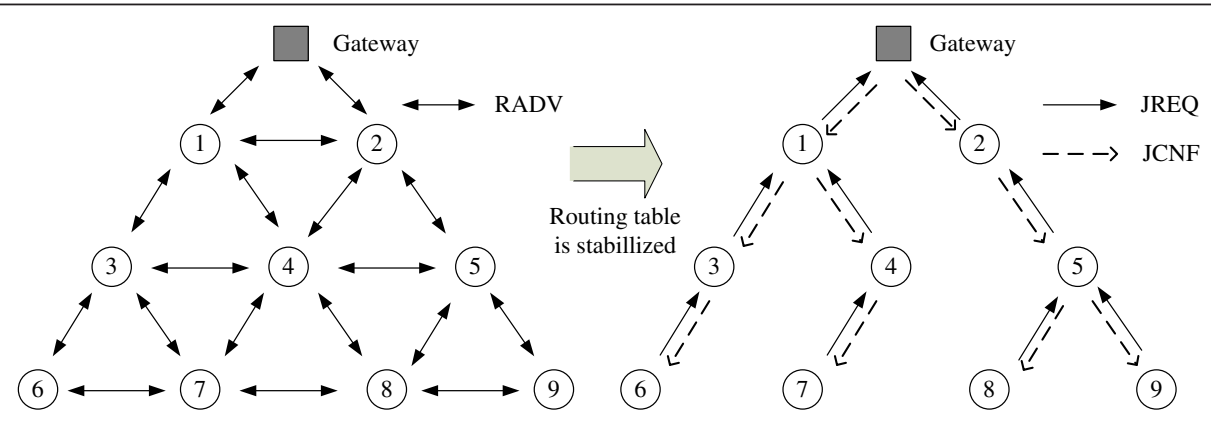

Route Advertisement (Neighbor Discovery)

Join and Slot Allocation

Figure 3 Example of route setup. 
subframe is divided into a number of time slots. Note that this subframe should be unique within the interference range to avoid collision in the neighboring area, but the number of available physical frequencies is limited by the physical layer (PHY) radio or external interference sources. To resolve this problem, we employ the multi-channel communication approach to extend the number of available subframes for supporting devices within the interference range. Consequently, the subframe can be defined by physical frequency and time indices. In our work, we used the IEEE 802.15.4 PHY radio, which allows for the use of 16 channels, where (16 $\times N)$ subframes are created. Accordingly, within the interference range of a specific node, $(16 \times N)$ nodes can be supported.

Each device in the network maintains its own subframe table, whose entries include the device ID, subframe information (i.e., a pair of frequency index and time index), hop count, and sequence number of each device within the interference range. Each device in the network randomly selects its own subframe among the selectable subframes and then broadcasts the CADV message by one hop, periodically, which includes the subframe table information. Whenever a device receives a CADV message, the device updates its subframe table. At this time, the device checks whether its subframe has been duplicated in another device. If it has been duplicated, the device with the larger ID value reselects its subframe, except for the duplicated one. This procedure continues until the subframe table of the device becomes stable. If all the entries of the subframe table do not change during a certain period of time, the device permanently sets the subframe. In the subsequent TDMA-based on/off phase, a device uses this subframe to communicate with its child devices. To communicate with its parent, a device uses its parent's subframe. Thus, a device should not have the same time index as its parent so that it can communicate with both its parent and its children.

\subsection{Join and route recovery}

When a new device is turned on initially, it waits for a HELLO message from neighbors. As shown in Figure 4, every device has FREE duration in its subframe. Note that the device has to operate in CSMA mode at the common physical channel and maintain active status in FREE duration. The device broadcasts a HELLO message by one hop in every FREE duration (i.e., a HELLO message is broadcasted periodically).

When the new device receives the HELLO message from a neighbor, the new device responds with the RSREQ message to ask for the route and subframe information. When the neighbor device receives the RSREQ from the new device, the neighbor device has to respond with the message including the route and subframe information. As a result, the new device is able to obtain the neighbor's route and subframe information and update its routing/subframe tables. Upon receiving a HELLO message, the new device can obtain its neighbor's channel and route information using this procedure. After all the table entries of the new node become stable, the joining procedure of route management is performed. Likewise, the subframe management procedure can be also performed.

The route recovery procedure is the same as the join case. If a link failure notification from a lower layer arrives, the device detects a transmission error and operates like a new node. However, in this case, because the device already knows its neighbor's subframe occupation state, it requests only the route information with the RREQ message, except for the subframe information.

\section{Prototype implementation}

\subsection{Implementation}

In this section, we present the implementation for the proposed framework. As shown in Figure 5, we developed the hardware prototype of M2M mote device and debugging board according to IEEE 802.15.4, which is considered a major technical candidate for capillary M2M networks. The device has a connector to extend the sensing modules, and it is composed of the Chipcon CC2420 RF chip [32] and the Texas Instruments MSP430 microcontroller (Dallas, Texas, USA) [33]. The CC2420 chip has a maximum data transmit rate of 250 Kbps and a receiver sensitivity of $-95 \mathrm{dBm}$ and operates in the 2.4-GHz bandwidth. Built on a 16-bit RISC CPU, the MSP430 has a 12-bit A/D converter. In addition, this $60 \times 32-\mathrm{mm}^{2}$ device has $116 \mathrm{~KB}$ of flash memory and 8 $\mathrm{KB}$ of RAM. A full networking stack including the proposed JRS framework described in Section 2 is implemented using nesC and the TinyOS 2.0 operating systems. Table 1 provides details about the hardware specification.

A miniature testbed for the application of the proposed framework was built for demonstration, as shown in Figure 6. To set up a multi-hop network within the limited space of a miniature building, we intentionally attenuated the transmission power of each device to its minimum value and increased the receiver sensitivity by software modification. In other words, the packet with a higher receiver signal strength indicator value than receiver sensitivity of hardware could be discarded if it is smaller than the value modified by software. With this approach, we constructed a multi-hop network consisting of 15 devices. Figure 7 shows the network topology. This testbed can be regarded as an instance of a simplified intelligent building system for the specific 


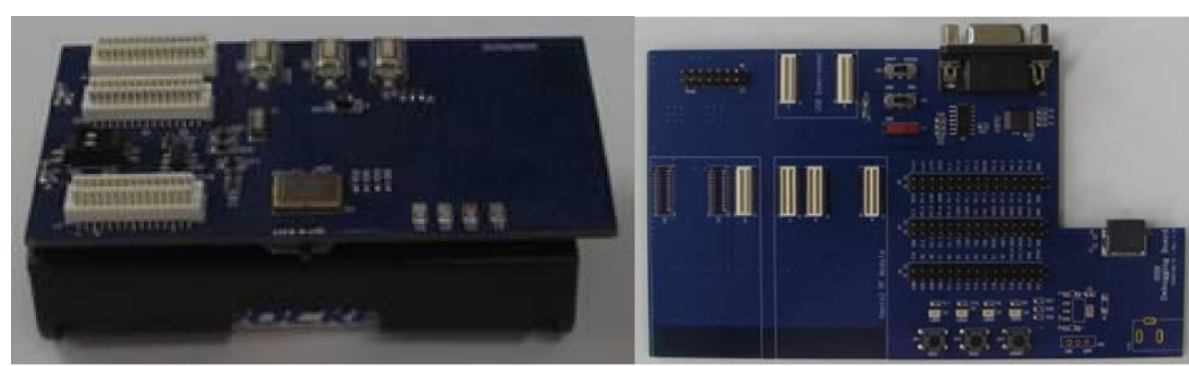

(a)
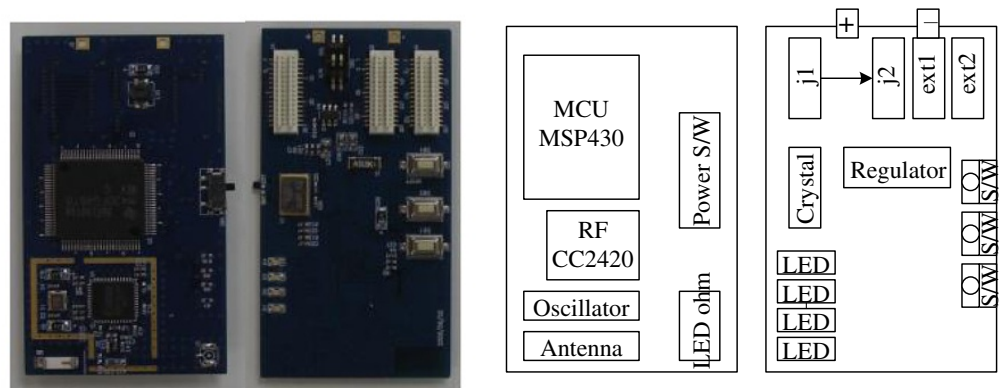

(b)

Figure 5 Hardware prototype. (a) IEEE 802.15.4-based device and debugging board. (b) Device layout (head and tail),

purpose of fire surveillance. A temperature sensor is attached to each device in the testbed. In our demonstration, when the temperature sensed at a device rises above a specific point, the sensing data are forwarded to the M2M gateway device, which then sends a notification about this event to a mobile phone through a dongle device. Then, we can observe the burning position on the three-dimensional visualization application of the mobile phone.

\subsection{Experiment and performance evaluation}

For the purpose of comparison, we also implemented a tree routing of ZigBee [34] and unslotted CSMA MAC in TinyOS 2.0 on our mote device. We evaluated the

\section{Table 1 Hardware specification}

\begin{tabular}{|c|c|c|}
\hline \multirow{2}{*}{$\begin{array}{l}\text { General } \\
\text { Features }\end{array}$} & Board size & $60 \times 32\left(\mathrm{~mm}^{2}\right)$ \\
\hline & operating system & TinyOS 2.0 \\
\hline \multirow[t]{4}{*}{ Processor } & Product number & MSP430FG4618 (Texas Instruments) \\
\hline & Memory & $116 \mathrm{~KB}$ of flash memory \\
\hline & & $8 \mathrm{~KB}$ of SRAM \\
\hline & Miscellaneous & 12-bit A/D, D/A converter \\
\hline \multirow[t]{6}{*}{ Radio } & Product number & CC2420 (Texas Instruments) \\
\hline & Frequency range & 2400 to approximately $2483.5(\mathrm{MHz})$ \\
\hline & Transmit data rate & 250 Kbps (Max.) \\
\hline & Transmit power & $0 \mathrm{dBm}$ \\
\hline & Receiver sensitivity & $-95 \mathrm{dBm}$ \\
\hline & Antenna & Chip antenna \\
\hline \multirow[t]{5}{*}{ Miscellaneous } & Temperature sensor & LM35DZ (NS) \\
\hline & Current consumption & 28 mA @ RF mode \\
\hline & & $470 \mu \mathrm{A} @$ Idle mode \\
\hline & Power supply & 1.5-V Alkaline battery $(\times 2)$ (AA Size) \\
\hline & Connectors & 30-Pin connectors for sensor board \\
\hline
\end{tabular}




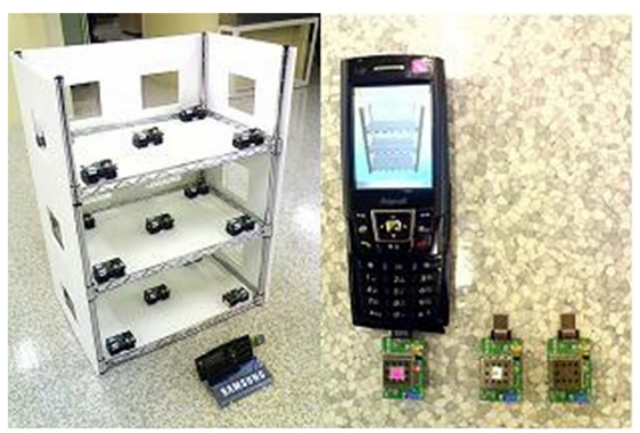

(a) (b)

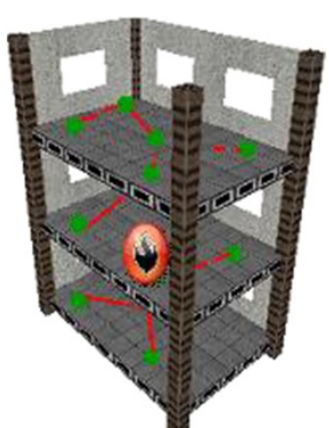

(c)

Figure 6 Miniature testbed setup. (a) Miniature testbed for a building fire-alarm system. (b) Mobile phone and dongle device for collecting data. (c) Three-dimensional visualization application of the miniature testbed on a mobile phone.

performance in terms of the following three metrics: the average packet reception rate, average per-hop delivery latency, and energy efficiency. For fair comparison in the experiments of the packet reception rate and the perhop delivery latency, we did not use the CSMA duty cycling; that is, all the devices operate in always-on status. In the experiments, we constructed a network topology consisting of 15 devices (Figure 7), in which all the devices except for the M2M gateway are data sources and the packets of 127 bytes are sent toward the predesignated M2M gateway. We controlled the packet generation rate (i.e., packets per device per second) to vary the traffic load in the network. We repeated each

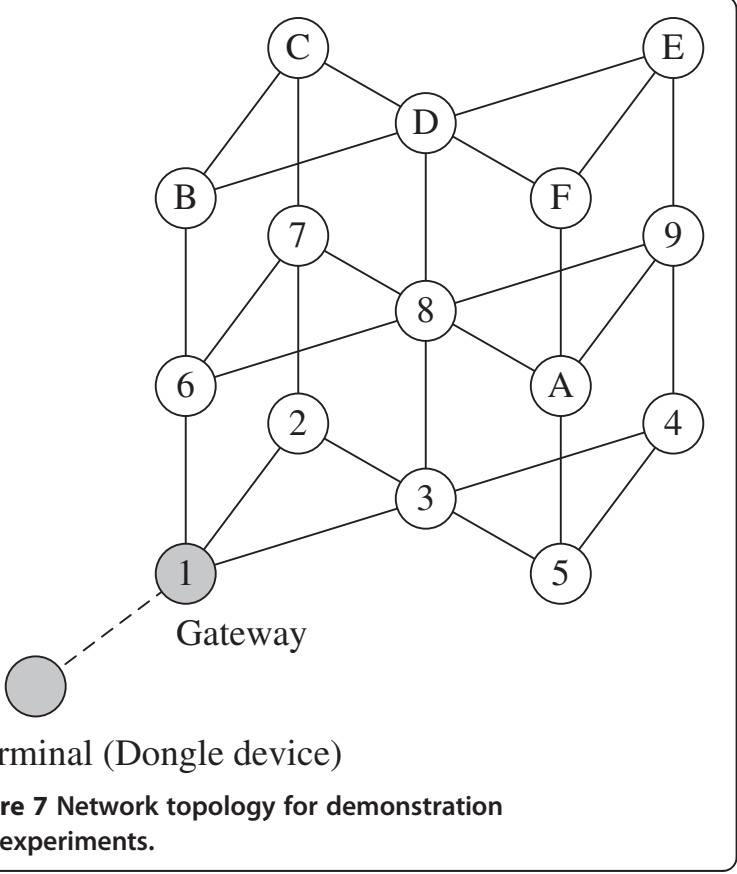

experiment 20 times to collect enough data for statistical confidence.

Figure 8 shows the average packet reception rate with increasing packet generation rate. Overall, JRS exhibits a significantly higher packet reception rate than the ZigBee tree routing. Under low traffic conditions (i.e., packet generation rate $<2.0$ ), both JRS and ZigBee achieved good reception rates. However, as the packet generation rate increases, the reception rate of ZigBee sharply deceases because of the high contention overhead of CSMA. In contrast, JRS maintains a high reception rate even under high traffic conditions.

We measured the average per-hop delivery latency with increasing packet generation rate (Figure 9). In the figure, the latency of ZigBee quickly increases as the traffic load increases. The reason is that the CSMA transmission of ZigBee requires a longer back-off time

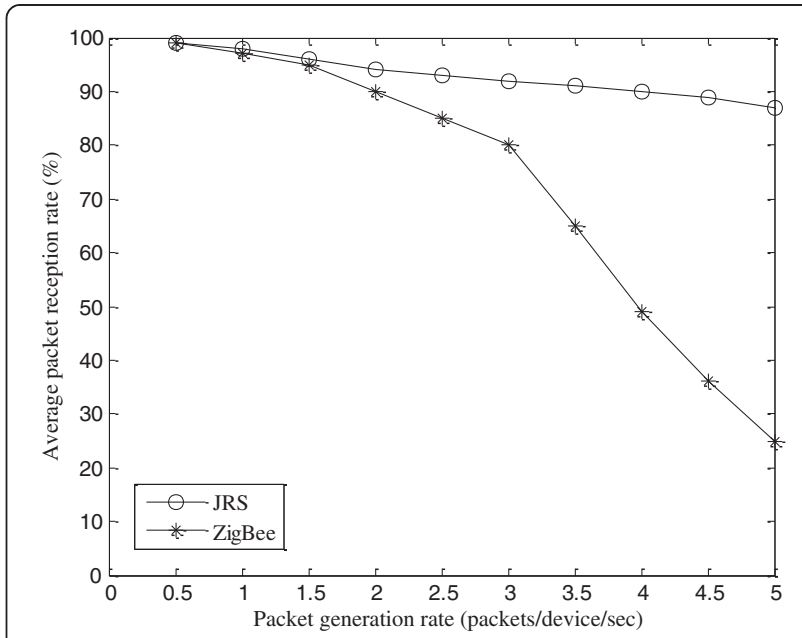

Figure 8 Average packet reception rate. 


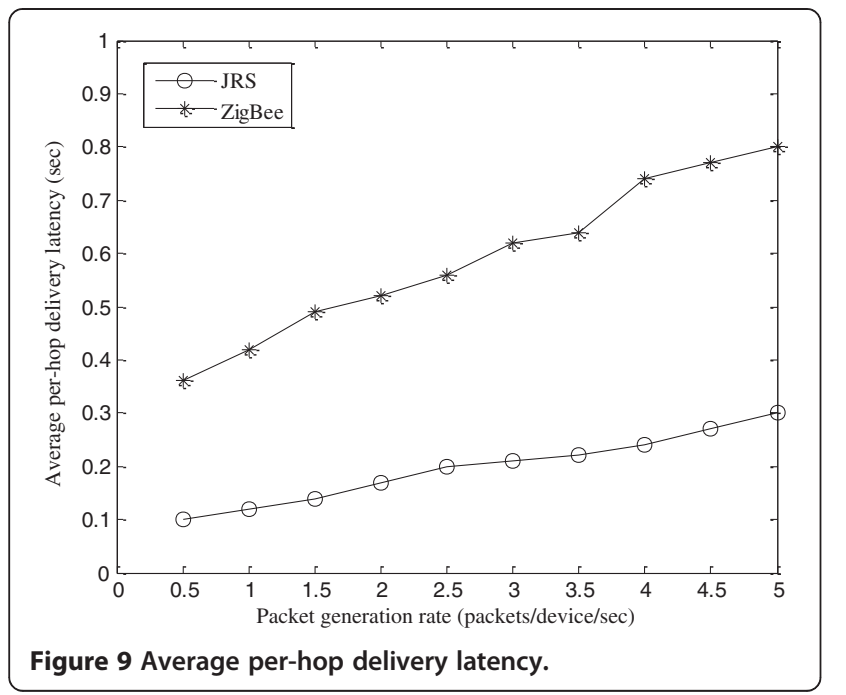

for resolving collisions under high traffic conditions. On the other hand, JRS uses TDMA transmission for data delivery, which does not require an additional waiting time such as random back-off. Moreover, JRS provides the scheduled concurrent transmissions of adjacent devices through a multi-channel approach, and thus, is able to maintain a certain level of per-hop latency even under heavy traffic conditions.

In the third experiment, we used the CSMA duty cycling on the ZigBee, which is being considered as a general solution for extending network lifetime, for the energy efficiency comparison. In this experiment, the measured values for the RF active current, idle current, and active time per packet are $28 \mathrm{~mA}, 470 \mu \mathrm{A}$, and 46.4 $\mathrm{ms}$, respectively. The results of this experiment are shown in Table 2. From the results, we can observe that the proposed framework exhibits more energy efficiency on average by $26 \%$.

In the existing CSMA duty-cycling approach, each device in the network has to wait for back-off time before transmitting its pending packets to avoid collision with neighbor devices. Moreover, the transceiver of each device has to maintain active status during the fixed portion of the beacon interval according to the

Table 2 Energy efficiency comparisons

\begin{tabular}{lccc}
\hline $\begin{array}{l}\text { Packet generation } \\
\text { rate }\end{array}$ & $\begin{array}{c}\text { Data rate } \\
\text { (Kbps) }\end{array}$ & $\begin{array}{c}\text { Current } \\
\text { consumption (mA) }\end{array}$ & $\begin{array}{c}\text { Reduction } \\
\text { rate (\%) }\end{array}$ \\
\hline $\begin{array}{l}\text { CSMA duty } \\
\text { cycling (10\%) }\end{array}$ & 1.5 & 3.22 & - \\
$\begin{array}{l}\text { JRS framework } \\
(1.5 \text { packets/s) }\end{array}$ & 1.5 & 2.39 & 26 \\
$\begin{array}{l}\text { CSMA duty } \\
\text { Cycling (20\%) }\end{array}$ & 3.1 & & - \\
JRS framework & & 5.98 & 28 \\
$(3$ packets/s) & 3.1 & 4.30 & \\
\hline
\end{tabular}

predefined duty-cycling rate. This approach causes an increase in latency under heavy traffic conditions and suffers from unnecessary energy waste caused by the relatively long active duration under light traffic conditions. On the other hand, in the proposed framework, the transceiver of each device remains active only when it has pending packets or is receiving data. In addition, there is no additional delay by the random back-off. In other words, the proposed framework could be an optimal solution to extend network lifetime under various traffic conditions.

\section{Conclusion}

JRS is a joint routing and scheduling framework for capillary M2M networks. To achieve the conflicting goals of maximizing network performance and conserving energy, JRS has the following important features: (1) CSMA-TDMA hybrid MAC approach to combine the strengths of both types of MAC protocols while offsetting their shortcomings; (2) integrated routing and MAC scheduling to minimize the protocol overhead, for which JRS organizes the multi-hop tree network through a route management procedure and assigns the subframe to each end device in a distributed manner; and (3) multi-channel communication to extend the number of subframes and enhance network performance. Experimental results with the prototype implementation demonstrate that JRS outperforms conventional schemes, even under heavy traffic conditions.

\section{Competing interests}

The authors declare that they have no competing interests.

\section{Authors' information}

TS received his Ph.D. degree in Information Security from Korea University, Seoul, Korea and his M.S. and B.S. degrees in Computer Engineering from Ajou University, Suwon, Korea. From August 2005 to February 2011, he was with the Samsung Electronics of Korea. Since 2011, he has been a professor at the Division of Information and Computer Engineering, Ajou University, Suwon, Korea. His research interests include convergence platform security, mobile cloud computing security, mobile/wireless network security, WPAN/ WSN security, anomaly detection algorithms, and machine learning applications.

HHC received his B.S., M.S., and Ph.D. degrees in Computer Science and Engineering from Sogang University, Seoul, Korea in 1994, 1996, and 2005, respectively. From 2005 to 2008, he was with the Samsung Electronics of Korea. Since 2009, he has been a professor at the Department of Computer Science, Inha Technical College, Incheon, Korea. His research interests include wireless sensor network, ubiquitous computing, and wireless mesh routing protocol.

EJK received his B.S., M.S., and Ph.D. degrees in Electronics and Computer Engineering from Korea University, Seoul, Korea in 2004, 2006, and 2013, respectively. From September 2005 to December 2005, he was with the Next Generation Wireless Communication (NGWC) Lab., Intel Korea R\&D Center at Intel Corporation. From February 2006 to July 2009, he was with the Digital Media \& Communications (DMC) R\&D Center at Samsung Electronics Co., Ltd. of Korea. Since August 2009, he has been with the Advanced Institute of Technology at KT Corporation, where he is currently a senior researcher. His research interests include wireless/mobile networks with an emphasis on QoS guarantee and adaptation, wireless sensor networks, wireless LAN/MAN/ 
PAN, next-generation mobile networks, and machine-to-machine communications.

\section{Acknowledgment}

This research was supported by Basic Science Research Program through the National Research Foundation of Korea (NRF) funded by the Ministry of Education, Science and Technology (no. 2012R1A1A1010667).

\section{Author details}

${ }^{1}$ Division of Information and Computer Engineering, Ajou University, Suwon 443-749, South Korea. ${ }^{2}$ Department of Computer Science, Inha Technical College, Incheon 402-752, South Korea. ${ }^{3}$ Advanced Institute of Technology, KT Corporation, Umyeon-dong, Seocho-gu, Seoul 137-792, South Korea.

Received: 26 January 2013 Accepted: 22 April 2013

Published: 10 May 2013

\section{References}

1. D Niyato, L Xiao, P Wang, Machine-to-machine communications for home energy management system in smart grid. IEEE Commun. Mag. 49(4), 53-59 (2011)

2. $R$ Lu, $X$ Li, $X$ Liang, $X$ Shen, $X$ Lin, GRS: the green, reliability, and security of emerging machine to machine communications. IEEE Commun. Mag. 49(4), 28-35 (2011)

3. Y Zhang, R Yu, S Xie, W Yao, Y Xiao, M Guizani, Home M2M networks: architectures, standards, and QoS improvement. IEEE Commun. Mag. 49(4), 44-52 (2011)

4. ZM Fadlullah, MM Fouda, N Kato, A Takeuchi, N Iwasaki, Y Nozaki, Toward intelligent machine-to-machine communications in smart grid. IEEE Commun. Mag. 49(4), 60-65 (2011)

5. G Wu, S Talwar, K Johnsson, N Himayat, KD Johnson, M2M: from mobile to embedded internet. IEEE Commun. Mag. 49(4), 36-43 (2011)

6. S-Y Lien, K-C Chen, Y Lin, Toward ubiquitous massive accesses in 3GPP machine-to-machine communications. IEEE Commun. Mag. 49(4), 66-74 (2011)

7. K Chang, A Soong, M Tseng, Z Xiang, Global wireless machine-to-machine standardization. IEEE Internet Comput. 15(2), 64-69 (2011)

8. Y Katsuhiko, S Hirohisa, Trends in M2M standardization and NEC's activities to promote the standardization of remote management technologies. NEC Tech. J. 6(4), 28-32 (2011)

9. ETSI TS 102690 v2.0.6, ETSI technical specification, machine-to-machine communications (M2M); functional architecture, 2012. http://portal.etsi.org

10. EJ Kim, S Youm, Machine-to-machine platform architecture for horizontal service integration. EURASIP Journal on Wireless Communications and Networking 2013(79), 1-9 (2013)

11. IEEE Std 802.15.4 2006, Wireless medium access control and physical layer specifications for low-rate wireless personal area networks. IEEE Computer Society (2006). http://ieeexplore.ieee.org

12. W Ye, J Heidemann, D Estrin, An energy-efficient MAC protocol for wireless sensor networks, in Proceedings of the 21st Annual Joint Conference of the IEEE Computer and Communications Societies INFOCOM'02 (IEEE, Washington, 2002, New York, 2002), pp. 1567-1576

13. TV Dam, K Langendoen, An adaptive energy-efficient MAC protocol for wireless sensor networks, in Proceedings of the 1st International Conference on Embedded Networked Sensor Systems SenSys'03 (ACM, New York, 2003, Los Angeles, 2003), pp. 171-180

14. J Hill, D Culler, Mica: a wireless platform for deeply embedded networks. IEEE Micro 22(6), 12-24 (2002)

15. AE Hoiydi, J-D Decotignie, MAC Wise, An ultra low power MAC protocol for multi-hop wireless sensor networks, in Proceedings of the 1st International Workshop on Algorithmic Aspects of Wireless Sensor Networks ALGOSENSORS'04 (ACM, New York, 2004, Turku, 2004), pp. 18-31

16. J Polastre, J Hill, D Culler, Versatile low power media access for wireless sensor networks, in Proceedings of the 2nd International Conference on Embedded Networked Sensor Systems SenSys'04 (ACM, New York, 2004, Baltimore, 2004), pp. 95-107

17. M Buettner, GV Yee, E Anderson, R Han, X-MAC: a short preamble MAC protocol for duty-cycled wireless sensor networks, in Proceedings of the 4th International Conference on Embedded Networked Sensor Systems SenSys'06 (ACM, New York, 2003, Boulder, 2006), pp. 307-320
18. SC Ergen, P Varaiya, PEDAMACS: power efficient and delay aware medium access protocol for sensor networks. IEEE Trans. Mobile Comput 5(7), 920-930 (2006)

19. LV Hoesel, P Havinga, A Lightweight Medium Access Protocol (LMAC) for Wireless Sensor Networks: Reducing Preamble Transmissions and Transceiver State Switches, in Proceeding of the 1st International Workshop on Networked Sensing Systems INSS'04 (ACM, New York, 2004, 2004), pp. 205-208

20. GP Halkes, KG Langendoen, Crankshaft: an energy-efficient MAC-protocol for dense wireless sensor networks, in Proceedings of the 4th European Conference on Wireless Sensor Networks EWSN'07 (ACM, New York, 2009, Delft, 2007), pp. 228-244

21. W Hu, X Li, H Yousefi'zadeh, LA-MAC: a load adaptive MAC protocol for MANETs, in Proceedings of the 28th IEEE Conference on Global Telecommunications GLOBECOM'09 (ACM, New York, 2009, Honolulu, 2009), pp. 1-6

22. P Bahl, R Chandra, J Dunagan, SSCH: Slotted seeded channel hopping for capacity improvement in IEEE 802.11 ad hoc wireless networks, in Proceedings of. the 10th Annual International Conference on Mobile Computing and Networking MobiCom'04 (ACM, New York, 2004, Philadelphia, 2004), pp. 216-230

23. N Jain, SR Das, A Nasipuri, A multichannel CSMA MAC protocol with receiver-based channel selection for multihop wireless networks, in Proceedings of the 10th International Conference on Computer Communications and Networks, IC3N'01 (ACM, New York, 2001, Scottsdale, Arizona, 2001), pp. 432-439

24. A Nasipuri, J Zhuang, SR Das, A multichannel CSMA MAC protocol for multihop wireless networks, in Proceeding of the IEEE Wireless Communications and Networking Conference WCNC'99 (IEEE, Washington, 1999, New Orleans, 1999), pp. 1402-1406

25. A Nasipuri, SR Das, A Nasipuri, SR Das, Multichannel CSMA with signal power-based channel selection for multihop wireless networks, in Proceedings of the 52nd Vehicular Technology Conference VTC'00 (IEEE, Washington, 2000, Boston, 2000), pp. 211-218

26. J So, NH Vaidya, Multi-channel MAC for ad hoc networks: Handling multichannel hidden terminals using a single transceiver, in Proceedings of the 5th ACM International Symposium on Mobile Ad Hoc Networking and Computing MOBIHOC'04 (ACM, New York, 2004, Tokyo, 2004), pp. 222-233

27. Z Tang, JJ Garcia-Luna-Aceves, Hop-Reservation Multiple Access (HRMA) for Ad-Hoc Networks, in Proceedings of the 21st Annual Joint Conference of the IEEE Computer and Communications Societies INFOCOM'99 (IEEE, Washington, 1999, New York, 1999), pp. 194-201

28. S Wu, C Lin, Y Tseng, J Sheu, A new multi-channel MAC protocol with ondemand channel assignment for multi-hop mobile ad hoc networks, in Proceedings of the 5th International Symposium on Parallel Architectures, Algorithms, and Networks I-SPAN'00 (Hindawi, New York, 2000, Dallas, 2000), pp. 232-237

29. R Draves, J Padhye, B Zill, Routing in multi-radio, multi-hop wireless mesh networks, in Proceedings of the 10th Annual International Conference on Mobile Computing and Networking MobiCom'04 (ACM, New York, 2004, Philadelphia, 2004), pp. 114-128

30. A Raniwala, K Gopalan, T Chiueh, Centralized channel assignment and routing algorithms for multi-channel wireless mesh networks. ACM SIGMOBILE Mobile Computing and Communications Review 8(2), 50-65 (2004)

31. TinyOS 2.0. http://www.tinyos.net. Accessed 7 Jan 2013

32. Chipcon, Chipcon Products from Texas Instruments. http://www.chipcon.com Accessed 7 Jan 2013

33. MSP430, Texas Instruments. http://www.ti.com. Accessed 7 Jan 2013

34. ZigBee Alliance, ZigBee Specification, Document 053474r13, 2006. http://www. zigbee.org

\section{doi:10.1186/1687-1499-2013-126}

Cite this article as: Shon et al: Joint routing and scheduling in multichannel capillary machine-to-machine networks. EURASIP Journal on Wireless Communications and Networking 2013 2013:126. 